\title{
A note on the Sobol' indices and interactive criteria
}

\author{
Michel GRABISCH${ }^{1 *}$ and Christophe LABREUCHE ${ }^{2}$ \\ ${ }^{1}$ Paris School of Economics, University of Paris I \\ 106-112, Bd de l'Hôpital, 75013 Paris, France \\ michel.grabisch@univ-paris1.fr \\ 2 Thales Research and Technology \\ 1, Avenue Augustin Fresnel, 91767 Palaiseau, France \\ christophe.labreuche@thalesgroup.com
}

\begin{abstract}
The Choquet integral and the Owen extension (or multilinear extension) are the most popular tools in multicriteria decision making to take into account the interaction between criteria. It is known that the interaction transform and the Banzhaf interaction transform arise as the average total variation of the Choquet integral and multilinear extension respectively. We consider in this note another approach to define interaction, by using the Sobol' indices which are related to the analysis of variance of a multivariate model. We prove that the Sobol' indices of the multilinear extension gives the square of the Fourier transform, a well-known concept in computer sciences. We also relate the latter to the Banzhaf interaction transform and compute the Sobol' indices for the 2-additive Choquet integral.
\end{abstract}

Keywords: capacity, interaction index, Sobol' index, multilinear extension, Fourier transform

\section{Introduction}

In multicriteria decision making, the Choquet integral with respect to a capacity has become a popular tool to model situations where some interaction exists between criteria [4]. This often happens in practice, as the evaluation of an alternative under several criteria is a complex process, where the level of importance of criteria generally depends on which criteria are satisfied or not.

The basic ingredient to model interaction is the capacity (or fuzzy measure) defined on the set $N$ of criteria, through its interaction transform [3], which is a generalization of the Shapley value. Another type of interaction has been introduced by Roubens [11] under the name Banzhaf interaction transform, and extends the Banzhaf value. So far, emphasis has been put on the former one in theoretical developments and applications.

It is well known that the Choquet integral is an extension of a capacity, seen as a pseudoBoolean function, and called the Lovász extension. Another popular extension of pseudoBoolean functions is the Owen extension or multilinear extension, known in Multi-Attribute Utility Theory from a long time ago [7]. Both can be considered as aggregation functions on a bounded closed domain (say, $[0,1]^{n}$ ). Defining the interaction index for $S \subseteq N$ w.r.t. an aggregation function $F$ on $[0,1]^{n}$ as the average of the total variation of $F$ w.r.t. the coordinates in $S$, Grabisch et al. [5] showed that the interaction transform corresponds to the interaction

\footnotetext{
${ }^{*}$ Corresponding author.
} 
index w.r.t. the Choquet integral, while the Banzhaf interaction transform is the interaction index w.r.t. the multilinear extension.

The present note aims to add another view of interaction to the picture, namely the statistical view, and to relate it to a well-known transform used in computer sciences but so far ignored in the field of decision making, which is the Fourier transform. We show that considering the aggregation model as a multivariate function and defining the interaction index of $S \subseteq N$ through the Sobol' index of $S$ (similar to the variance), we come up with the square of the Fourier transform when the aggregation function is the multilinear model (Theorem 1). We also show the close relation between the Fourier transform and the Banzhaf interaction transform, and compute the Sobol' indices for the 2-additive Choquet integral (Theorem 2).

Throughout the paper, cardinalities of sets $S, T, \ldots$ are denoted by the corresponding small letters $s, t, \ldots$.

\section{Basic notions}

We consider throughout a finite set $N=\{1, \ldots, n\}$. We often denote cardinality of sets $S, T, \ldots$ by corresponding small letters $s, t, \ldots$.

A set function is a mapping $\xi: 2^{N} \rightarrow \mathbb{R}$. A game $v$ is set function vanishing on the empty set: $v(\varnothing)=0$. A capacity [1] or fuzzy measure [14] $\mu$ is a game satisfying monotonicity: for every $S, T \in 2^{N}$ such that $S \subseteq T$, we have $\mu(S) \leqslant \mu(T)$.

Clearly, the set $\mathbb{R}^{2^{N}}$ of set functions on $N$ is a $2^{n}$-dimensional vector space. We introduce on $\mathbb{R}^{2^{N}}$ the following inner product:

$$
\left\langle\xi, \xi^{\prime}\right\rangle=\frac{1}{2^{n}} \sum_{S \subseteq N} \xi(S) \xi^{\prime}(S) .
$$

A classical basis of $\mathbb{R}^{2^{N}}$ is the basis of the unanimity games. For any nonempty subset $S \subseteq N$, the unanimity game centered on $S$ is the game defined by

$$
u_{S}(T)=\left\{\begin{array}{ll}
1, & \text { if } T \supseteq S \\
0, & \text { otherwise }
\end{array} .\right.
$$

Defining the set function $u_{\varnothing}(S)=1$ for every $S \subseteq N$, we obtain a basis of set functions. A drawback of this basis is that it is not orthogonal w.r.t. the above inner product. We will introduce later an orthonormal basis.

It is well known that the coordinates of $\xi$ in this basis are the Möbius transform coefficients:

$$
\xi=\sum_{S \in 2^{N}} m^{\xi}(S) u_{S}
$$

with

$$
m^{\xi}(S)=\sum_{T \subseteq S}(-1)^{|S \backslash T|} \xi(T) .
$$

The Möbius transform (or Möbius inverse) is a fundamental notion in combinatorics (see, e.g., [10]).

There is another vision of set functions, namely the pseudo-Boolean functions [6], noting that any subset $A$ of $N$ can be encoded by its characteristic function $1_{A}$. Formally, a pseudoBoolean function is a mapping $f:\{0,1\}^{n} \rightarrow \mathbb{R}$. If follows that the set of pseudo-Boolean functions of $n$ variables is a $2^{n}$-dimensional vector space, with inner product

$$
\left\langle f, f^{\prime}\right\rangle=\frac{1}{2^{n}} \sum_{x \in\{0,1\}^{n}} f(x) f^{\prime}(x) .
$$


The standard polynomial expression of a pseudo-Boolean function $f$ is

$$
f(x)=\sum_{A \subseteq N} f\left(1_{A}\right) \prod_{i \in A} x_{i} \prod_{i \in A^{c}}\left(1-x_{i}\right)
$$

for every $x \in\{0,1\}^{n}$, and with the convention $\prod_{i \in \varnothing} x_{i}=1$. Rearranging terms, we get a sum of monomials:

$$
f(x)=\sum_{T \subseteq N} a_{T} \prod_{i \in T} x_{i}
$$

for every $x \in\{0,1\}^{n}$, where the coefficients $a_{T}$ form the Möbius transform of $\xi_{f}$, the set function associated to $f$. Indeed, observe that unanimity games $u_{S}$ correspond to monomials $\prod_{i \in S} x_{i}$.

Starting from (2), the Owen extension [9] or multilinear extension is obtained by letting $x$ vary in $[0,1]^{N}$ :

$$
f^{\mathrm{Ow}}(x)=\sum_{T \subseteq N} a_{T} \prod_{i \in T} x_{i} \quad\left(x \in[0,1]^{N}\right) .
$$

Another possible extension is obtained by remarking that in (21), the product can be replaced by the minimum without changing the value of the function. Letting again $x$ to vary in $[0,1]^{N}$, we obtain the Lovász extension [8]:

$$
f^{\mathrm{Lo}}(x)=\sum_{T \subseteq N} a_{T} \bigwedge_{i \in T} x_{i} \quad\left(x \in[0,1]^{N}\right) .
$$

The Lovász extension coincides in fact with the Choquet integral [1].

\section{Transforms of set functions}

The Möbius transform presented above is an example of a linear and invertible transform on the set of set functions, in the sense that to each set function $\xi$ is associated another set function $m^{\xi}$, called the Möbius transform of $\xi$, being linear because $m^{\xi+\alpha \xi^{\prime}}=m^{\xi}+\alpha m^{\xi^{\prime}}$ for every $\xi, \xi^{\prime}$ and $\alpha \in \mathbb{R}$, and $\xi$ can be recovered from $m^{\xi}$ by the inverse transform:

$$
\xi(S)=\sum_{T \subseteq S} m^{\xi}(T) \quad\left(S \in 2^{N}\right)
$$

\subsection{Interaction transforms}

Other such transforms exist, and three of them are of importance in this paper. The first one is the interaction transform [3], defined by

$$
I^{\xi}(S)=\sum_{T \subseteq N \backslash S} \frac{(n-t-s) ! t !}{(n-s+1) !} \sum_{L \subseteq S}(-1)^{|S \backslash L|} \xi(T \cup L),
$$

and the inverse relation is given by

$$
\xi(S)=\sum_{K \subseteq N} \beta_{|S \cap K|}^{|K|} I^{\xi}(K)
$$

where

$$
\beta_{k}^{l}=\sum_{j=0}^{k}\left(\begin{array}{c}
k \\
j
\end{array}\right) B_{l-j} \quad(k \leq l)
$$


and $B_{0}, B_{1}, \ldots$ are the Bernoulli numbers. Also, the interaction transform has a simple expression in terms of the Möbius transform:

$$
I^{\xi}(S)=\sum_{T \supseteq S} \frac{1}{t-s+1} m^{\xi}(T) .
$$

The interaction transform is of primary importance in multicriteria decision making, as it permits to model interaction between criteria [4].

A similar transform is the Banzhaf interaction transform [11] defined by

$$
I_{\mathrm{B}}^{\xi}(S)=\left(\frac{1}{2}\right)^{n-s} \sum_{K \subseteq N}(-1)^{|S \backslash K|} \xi(K),
$$

with inverse relation

$$
\left(I_{\mathrm{B}}^{-1}\right)^{\xi}(S)=\sum_{K \subseteq N}\left(\frac{1}{2}\right)^{k}(-1)^{|K \backslash S|} \xi(K) .
$$

Its expression in terms of the Möbius transform is

$$
I_{\mathrm{B}}^{\xi}(S)=\sum_{K \supseteq S}\left(\frac{1}{2}\right)^{|K \backslash S|} m^{\xi}(K),
$$

and the converse relation is

$$
m^{\xi}(S)=\sum_{K \supseteq S}\left(-\frac{1}{2}\right)^{|K \backslash S|} I_{\mathrm{B}}^{\xi}(K) .
$$

In [5], the following close relation between the two extensions of a pseudo-Boolean function and the two interaction indices are shown:

$$
\begin{aligned}
I_{\mathrm{B}}^{\xi}(S) & =\int_{[0,1]^{n}} \frac{\partial^{s} f^{\mathrm{Ow}}}{\partial x_{S}}(x) d x \\
I^{\xi}(S) & =\int_{[0,1]^{n}} \Delta_{S} f^{\mathrm{Lo}}(x) d x
\end{aligned}
$$

where $f$ is the pseudo-Boolean function corresponding to $\xi, x_{S}$ is the restriction of $x$ to coordinates in $S$, and

$$
\Delta_{S} f^{\mathrm{Lo}}(x)=\sum_{T \supseteq S} m^{\xi}(T) \bigwedge_{i \in T \backslash S} x_{i},
$$

which plays the role of a partial derivative.

\subsection{Fourier transform}

Another transform is the Fourier transform, well known in computer sciences. It is defined as the coordinates of a set function in the basis of the parity functions. For any subset $S \subseteq N$, the parity function associated to $S$ is the function

$$
\chi_{S}(x)=(-1)^{1_{S} \cdot x}=(-1)^{\sum_{i \in S} x_{i}} \quad\left(x \in\{0,1\}^{n}\right),
$$

where $1_{S} \cdot x$ is the inner product between the two vectors $1_{S}, x$. The parity function outputs 1 if the number of variables in $S$ having value 1 is even, and -1 if it is odd. In terms of set functions, the parity function reads

$$
\chi_{S}(T)=(-1)^{|S \cap T|} \quad\left(T \in 2^{N}\right) .
$$


It can be checked that the set of parity functions forms an orthonormal basis of $\mathbb{R}^{2^{N}}$.

Let us denote by $\widehat{f}(S), S \subseteq N$, the coordinates of a pseudo-Boolean function $f$ in the basis of parity functions:

$$
f=\sum_{S \subseteq N} \widehat{f}(S) \chi_{S}
$$

The basis being orthonormal, it follows from (11) that $\widehat{f}(S)$ is simply given by

$$
\widehat{f}(S)=\left\langle f, \chi_{S}\right\rangle=\frac{1}{2^{n}} \sum_{x \in\{0,1\}^{n}}(-1)^{1_{S} \cdot x} f(x) \quad(S \subseteq N),
$$

or, in terms of set functions,

$$
\widehat{\xi}(S)=\frac{1}{2^{n}} \sum_{T \subseteq N}(-1)^{|S \cap T|} \xi(T) \quad(S \subseteq N) .
$$

The set of coordinates $\{\widehat{f}(S)\}_{S \subseteq N}$ is the Fourier transform.

We establish the relation between the Fourier, Möbius and Banzhaf transforms. Taking any set function $\xi$, we have

$$
\begin{aligned}
\widehat{\xi}(S) & =\frac{1}{2^{n}} \sum_{T \subseteq N}(-1)^{|S \cap T|} \xi(T) \\
& =\frac{1}{2^{n}} \sum_{T \subseteq N}(-1)^{|S \cap T|} \sum_{K \subseteq T} m^{\xi}(K) \\
& =\frac{1}{2^{n}} \sum_{K \subseteq N} m^{\xi}(K) \sum_{T \supseteq K}(-1)^{|S \cap T|} .
\end{aligned}
$$

Now,

$$
\begin{aligned}
\sum_{T \supseteq K}(-1)^{|S \cap T|}= & (-1)^{|K \cap S|} 2^{n-|K \cup S|}+(-1)^{|K \cap S|+1} 2^{n-|K \cup S|}\left(\begin{array}{c}
|S \backslash K| \\
1
\end{array}\right)+ \\
& (-1)^{|K \cap S|+2} 2^{n-|K \cup S|}\left(\begin{array}{c}
|S \backslash K| \\
2
\end{array}\right)+\cdots+(-1)^{|S|} 2^{n-|K \cup S|} \\
= & (-1)^{|K \cap S|} 2^{n-|K \cup S|}\left(1-\left(\begin{array}{c}
|S \backslash K| \\
1
\end{array}\right)+\left(\begin{array}{c}
|S \backslash K| \\
2
\end{array}\right)+\cdots+(-1)^{|S \backslash K|}\right) .
\end{aligned}
$$

Observe that

$$
1-\left(\begin{array}{c}
|S \backslash K| \\
1
\end{array}\right)+\left(\begin{array}{c}
|S \backslash K| \\
2
\end{array}\right)+\cdots+(-1)^{|S \backslash K|}=0
$$

except if $|S \backslash K|=0$. It follows that

$$
\begin{aligned}
\widehat{\xi}(S) & =\frac{1}{2^{n}} \sum_{K \supseteq S} m^{\xi}(K)(-1)^{|K \cap S|} 2^{n-|K \cup S|} \\
& =(-1)^{|S|} \sum_{K \supseteq S} \frac{1}{2^{k}} m^{\xi}(K) .
\end{aligned}
$$

Now, using ([6), we obtain

$$
\widehat{\xi}(S)=\left(\frac{-1}{2}\right)^{s} I_{\mathrm{B}}^{\xi}(S) .
$$

Lastly, we obtain from (15) and (7)

$$
m^{\xi}(S)=(-2)^{s} \sum_{T \supseteq S} \widehat{\xi}(T) .
$$




\section{The Sobol' indices}

In statistics, the analysis of variance (ANOVA) (see, e.g., Fischer and Mackenzie [2]) is a well-known tool to model interaction between variables in a multivariate model. Consider $n$ independent random variables $Z_{1}, \ldots, Z_{n}$, with uniform distribution on [0,1], and a multivariate model $Y=F(Z)$, where $Z=\left(Z_{1}, \ldots, Z_{n}\right)$. Let us denote for simplicity groups of variables $\left(Z_{i}\right)_{i \in S}$ by $Z_{S}$, and $Z_{-S}$ denotes $\left(Z_{i}\right)_{i \notin S}$. Hence, we may write $Z=\left(Z_{S}, Z_{-S}\right)$. Moreover, we denote by $\mathbb{E}[Y]$ the expected value of $Y$ taken over all variables $Z_{1}, \ldots, Z_{n}$. The expected value of $Y$ can be taken on a subset $Z_{S}$ of variables, with the corresponding notation $\mathbb{E}_{Z_{S}}[Y]$.

Any multivariate function can be decomposed in the following way (ANOVA decomposition):

$$
Y=F(Z)=F_{\varnothing}+\sum_{i=1}^{n} F_{i}\left(Z_{i}\right)+\sum_{i<j} F_{i j}\left(Z_{i}, Z_{j}\right)+\cdots+F_{N}(Z)=\sum_{S \subseteq N} F_{S}\left(Z_{S}\right),
$$

with

$$
\begin{aligned}
F_{\varnothing} & =\mathbb{E}[Y] \\
F_{i}\left(Z_{i}\right) & =\mathbb{E}\left[Y \mid Z_{i}\right]-F_{\varnothing} \\
F_{i j}\left(Z_{i}, Z_{j}\right) & =\mathbb{E}\left[Y \mid Z_{i}, Z_{j}\right]-F_{i}\left(Z_{i}\right)-F_{j}\left(Z_{j}\right)-F_{\varnothing} \\
& =\mathbb{E}\left[Y \mid Z_{i}, Z_{j}\right]-\mathbb{E}\left[Y \mid Z_{i}\right]-\mathbb{E}\left[Y \mid Z_{j}\right]+E[Y] \\
\vdots & =\vdots \\
F_{S}\left(Z_{S}\right) & =\mathbb{E}_{Z_{-S}}\left[Y \mid Z_{S}\right]-\sum_{T \subset S} F_{T}\left(Z_{T}\right)=\sum_{T \subseteq S}(-1)^{|S \backslash T|} \mathbb{E}_{Z_{-T}}\left[Y \mid Z_{T}\right] \\
& =\vdots \\
F_{N}(Z) & =\sum_{T \subseteq N}(-1)^{|N \backslash T|} \mathbb{E}_{Z_{-T}}\left[Y \mid Z_{T}\right] .
\end{aligned}
$$

The property of this decomposition is that each term has zero mean, except the first one, $F_{\varnothing}$. It follows that the variance of $Y$ can be decomposed as follows:

$$
\operatorname{Var}[Y]=\sum_{\varnothing \neq S \subseteq N} \operatorname{Var}\left[F_{S}\left(Z_{S}\right)\right]
$$

The first-order Sobol' indices [12, 13] are the quantities $\frac{\operatorname{Var}\left[F_{S}\left(Z_{S}\right)\right]}{\operatorname{Var}[Y]}$, although one can omit the normalization factor. The next theorem establishes the close link between Sobol' indices and the Fourier transform (and consequently the Banzhaf transform) for the multilinear model.

Theorem 1. Consider the multilinear extension $f_{\mu}^{\mathrm{Ow}}$ of a capacity $\mu$. Then the (nonnormalized) Sobol' index for a subset $\varnothing \neq S \subseteq N$ is given by

$$
\operatorname{Var}\left[\left(f_{\mu}^{\mathrm{Ow}}\right)_{S}\right]=\frac{1}{3^{s}}(\widehat{\mu}(S))^{2},
$$

where $\widehat{\mu}$ is the Fourier transform of $\mu$. Moreover, the ANOVA decomposition takes the following form

$$
f_{\mu}^{\mathrm{Ow}}(x)=\sum_{S \subseteq N}(-1)^{s} \prod_{i \in S}\left(2 x_{i}-1\right) \times \widehat{\mu}(S) .
$$


Proof. We set for simplicity $f=f_{\mu}^{\mathrm{Ow}}$. We compute

$$
f_{S}=\sum_{K \subseteq S}(-1)^{k} \mathbb{E}\left(f \mid Z_{S \backslash K}\right) \quad(S \subseteq N,|S|>0) .
$$

We have for any such $S$ :

$$
\begin{aligned}
\mathbb{E}\left(f \mid Z_{S \backslash K}\right) & =\int_{[0,1]^{N \backslash(S \backslash K)}} f \mathrm{~d} z_{N \backslash(S \backslash K)}=\sum_{T \subseteq N} m^{\mu}(T) \int_{[0,1]^{N \backslash(S \backslash K)}} \prod_{i \in T} z_{i} \mathrm{~d} z_{N \backslash(S \backslash K)} \\
& =\sum_{T \subseteq N} m^{\mu}(T) \frac{1}{2^{|T \backslash(S \backslash K)|}} \prod_{i \in T \cap(S \backslash K)} z_{i} \\
& =\sum_{L \subseteq N \backslash S} \sum_{T \subseteq S} m^{\mu}(L \cup T) \frac{1}{2^{|L \cup(T \cap K)|}} \prod_{i \in T \backslash K} z_{i} .
\end{aligned}
$$

It follows that

$$
\begin{aligned}
f_{S} & =\sum_{K \subseteq S}(-1)^{k} \sum_{L \subseteq N \backslash S} \sum_{T \subseteq S} m^{\mu}(L \cup T) \frac{1}{2^{|L \cup(T \cap K)|}} \prod_{i \in T \backslash K} z_{i} \\
& =\sum_{L \subseteq N \backslash S} \frac{1}{2^{l}} \sum_{T \subseteq S} m^{\mu}(L \cup T) \sum_{K \subseteq S}(-1)^{k} \frac{1}{2^{|T \cap K|}} \prod_{i \in T \backslash K} z_{i} .
\end{aligned}
$$

Letting $T^{\prime}=T \cap K$, we have

$$
\sum_{K \subseteq S}(-1)^{k} \frac{1}{2^{|T \cap K|}} \prod_{i \in T \backslash K} z_{i}=\sum_{T^{\prime} \subseteq T}(-1)^{t^{\prime}} \frac{1}{2^{t^{\prime}}} \prod_{i \in T \backslash T^{\prime}} z_{i} \sum_{K^{\prime} \subseteq S \backslash T}(-1)^{k^{\prime}} .
$$

Observing that $\sum_{K^{\prime} \subseteq S \backslash T}(-1)^{k^{\prime}}=0$ except if $S \backslash T=\emptyset$, it follows that

$$
f_{S}=\sum_{L \subseteq N \backslash S} \frac{1}{2^{l}} m^{\mu}(L \cup S) \sum_{T \subseteq S}(-1)^{t} \frac{1}{2^{t}} \prod_{i \in S \backslash T} z_{i} .
$$

Observe that

$$
\sum_{T \subseteq S}(-1)^{t} \frac{1}{2^{t}} \prod_{i \in S \backslash T} z_{i}=\frac{1}{2^{s}} \prod_{i \in S}\left(2 z_{i}-1\right),
$$

hence we finally get by (14):

$$
f_{S}=(-1)^{s} \prod_{i \in S}\left(2 z_{i}-1\right) \widehat{\mu}(S) .
$$

Note that this expression is also true for $S=\emptyset$ as

$$
f_{\emptyset}=\int_{[0,1]^{N}} f(z) \mathrm{d} z=\sum_{T \subseteq N} m^{\mu}(T) \int_{[0,1]^{N}} \prod_{i \in T} z_{i} \mathrm{~d} z=\sum_{T \subseteq N} \frac{m^{\mu}(T)}{2^{t}}=\widehat{\mu}(\emptyset) .
$$

We obtain finally

$$
\mathbb{E}\left[f_{S}^{2}\right]=\int_{[0,1]^{S}}\left(\prod_{i \in S}\left(2 z_{i}-1\right) \widehat{\mu}(S)\right)^{2} \mathrm{~d} z_{S}=\frac{1}{3^{s}}(\widehat{\mu}(S))^{2} .
$$


This result is not very surprising as, historically, Sobol' generalized the Fourier base to obtain the decomposition underlying the Sobol' indices [13].

Therefore, up to a multiplicative constant depending on the cardinality of the subset, the Sobol' indices are the coefficients of the square of the Fourier transform, or, due to (15), of the square of the Banzhaf transform (compare with (8)) .

The computation of the Sobol' indices for a general Choquet integral is quite complex due to the presence of the minimum operator in (3), and there does not seem to be a compact and appealing expression. We restrict ourself to a sub-class of capacities - called 2 additive - where all Möbius coefficient of cardinality strictly greater than 2 are zero [3]. The Choquet integral of such capacities therefore becomes (see (3))

$$
f^{\mathrm{Lo}}(z)=\sum_{i \in N} m(i) z_{i}+\sum_{\{i, j\} \subseteq N} m(i, j) z_{i} \wedge z_{j}
$$

with the notation $m(i)=m(\{i\})$ and $m(i, j)=m(\{i, j\})$, the Möbius transform of the capacity.

Theorem 2. Consider the Lovász extension $f_{\mu}^{\mathrm{Lo}}$ of a 2-additive capacity $\mu$ with Möbius transform $m$. Then $f_{\mu}^{\mathrm{Lo}}$ is decomposed in the following terms according to ANOVA

$$
\begin{aligned}
& f_{\emptyset}^{\mathrm{Lo}}=\sum_{i \in N} \frac{m(i)}{2}+\sum_{\{i, j\} \subseteq N} \frac{m(i, j)}{3} \\
& f_{k}^{\mathrm{Lo}}\left(z_{k}\right)=-\frac{m(k, \cdot)}{2} z_{k}^{2}+(m(k)+m(k, \cdot)) z_{k}-\left(\frac{m(k)}{2}+\frac{m(k, \cdot)}{3}\right) \\
& f_{p, q}^{\mathrm{Lo}}\left(z_{p}, z_{q}\right)=m(p, q)\left(-z_{p} \vee z_{q}+\frac{z_{p}{ }^{2}}{2}+\frac{z_{q}{ }^{2}}{2}+\frac{1}{3}\right)
\end{aligned}
$$

where $m(k, \cdot):=\sum_{i \in N \backslash\{k\}} m(k, i)$. Moreover, the (nonnormalized) Sobol' index are given by

$$
\begin{aligned}
& \operatorname{Var}\left[f_{k}^{\mathrm{Lo}}\right]=\frac{m(k)^{2}}{12}+\frac{m(k, \cdot)^{2}}{45}+\frac{m(k) m(k, \cdot)}{12} \\
& \operatorname{Var}\left[f_{p, q}^{\mathrm{Lo}}\right]=\frac{m(p, q)^{2}}{90}
\end{aligned}
$$

Proof.

$$
f_{\emptyset}^{\mathrm{Lo}}=\sum_{i \in N} m(i) \mathbb{E}\left[Z_{i}\right]+\sum_{\{i, j\} \subseteq N} m(i, j) \mathbb{E}\left[Z_{i} \wedge Z_{j}\right]
$$

where $\mathbb{E}\left[Z_{i} \wedge Z_{j}\right]=2 \int_{0}^{1} \int_{0}^{z_{i}} z_{i} \wedge z_{j} d z_{i} d z_{j}=2 \int_{0}^{1} \int_{0}^{z_{i}} z_{j} d z_{i} d z_{j}=2 \int_{0}^{1} \frac{z_{i}^{2}}{2} d z_{i}=\frac{1}{3}$. Hence (20) is proved.

For $k \in N$,

$$
f_{k}^{\mathrm{Lo}}\left(z_{k}\right)=\sum_{i \in N} m(i)\left(\mathbb{E}\left[Z_{i} \mid Z_{k}=z_{k}\right]-\frac{1}{2}\right)+\sum_{\{i, j\} \subseteq N} m(i, j)\left(\mathbb{E}\left[Z_{i} \wedge Z_{j} \mid Z_{k}=z_{k}\right]-\frac{1}{3}\right)
$$

We observe that $\mathbb{E}\left[Z_{k} \wedge Z_{i} \mid Z_{k}=z_{k}\right]=\int_{0}^{1} z_{k} \wedge z_{i} d z_{i}=\int_{0}^{z_{k}} z_{i} d z_{i}+\int_{z_{k}}^{1} z_{k} d z_{i}=z_{k}-\frac{z_{k}^{2}}{2}$. Hence

$$
\begin{aligned}
f_{k}^{\mathrm{Lo}}\left(z_{k}\right) & =m(k)\left(z_{k}-\frac{1}{2}\right)+m(k, \cdot)\left(z_{k}-\frac{z_{k}^{2}}{2}-\frac{1}{3}\right) \\
& =-\frac{m(k, \cdot)}{2} z_{k}^{2}+(m(k)+m(k, \cdot)) z_{k}-\left(\frac{m(k)}{2}+\frac{m(k, \cdot)}{3}\right) .
\end{aligned}
$$


Hence (21) is proved. Then

$$
\begin{aligned}
\mathbb{E}\left[\left(f_{k}^{\mathrm{Lo}}\right)^{2}\right] & =\int_{0}^{1}\left[m(k)^{2}\left(z_{k}^{2}-z_{k}+\frac{1}{4}\right)+m(k, \cdot)^{2}\left(z_{k}^{2}+\frac{z_{k}^{4}}{4}+\frac{1}{9}-z_{k}{ }^{3}-\frac{2 z_{k}}{3}+\frac{z_{k}{ }^{2}}{3}\right)\right. \\
& \left.+2 m(k) m(k, \cdot)\left(z_{k}^{2}-\frac{z_{k}{ }^{3}}{2}-\frac{z_{k}}{3}-\frac{z_{k}}{2}+\frac{z_{k}{ }^{2}}{4}+\frac{1}{6}\right)\right] d z_{k} \\
& =\frac{m(k)^{2}}{12}+\frac{m(k, \cdot)^{2}}{45}+\frac{m(k) m(k, \cdot)}{12}
\end{aligned}
$$

Hence (23) holds.

Consider now

$$
\begin{aligned}
f_{p, q}^{\mathrm{Lo}}\left(z_{p}, z_{q}\right) & =m(p, q)\left(z_{p} \wedge z_{q}-\mathbb{E}\left[Z_{p} \wedge Z_{q} \mid Z_{p}=z_{p}\right]-\mathbb{E}\left[Z_{p} \wedge Z_{q} \mid Z_{q}=z_{q}\right]-\mathbb{E}\left[Z_{p} \wedge Z_{q}\right]\right) \\
& =m(p, q)\left(z_{p} \wedge z_{q}-\left(z_{p}-\frac{z_{p}^{2}}{2}\right)-\left(z_{q}-\frac{z_{q}^{2}}{2}\right)+\frac{1}{3}\right) \\
& =m(p, q)\left(-z_{p} \vee z_{q}+\frac{z_{p}^{2}}{2}+\frac{z_{q}^{2}}{2}+\frac{1}{3}\right)
\end{aligned}
$$

as $z_{p} \wedge z_{q}+z_{p} \vee z_{q}=z_{p}+z_{q}$. Hence (22) is proved. Then

$$
\begin{aligned}
& \mathbb{E}\left[\left(f_{p, q}^{\mathrm{Lo}}\right)^{2}\right]=m(p, q)^{2} \int_{0}^{1} \int_{0}^{1}\left(-z_{p} \vee z_{q}+\frac{z_{p}^{2}}{2}+\frac{z_{q}^{2}}{2}+\frac{1}{3}\right)^{2} d z_{p} d z_{q} \\
& =m(p, q)^{2} \int_{0}^{1} \int_{0}^{1}\left(\left(z_{p} \vee z_{q}\right)^{2}-\left(z_{p} \vee z_{q}\right)\left(z_{p}^{2}+z_{q}^{2}+\frac{2}{3}\right)\right) d z_{p} d z_{q} \\
& +m(p, q)^{2} \int_{0}^{1} \int_{0}^{1}\left(\frac{z_{p}{ }^{4}}{4}+\frac{z_{q}{ }^{4}}{4}+\frac{z_{p}{ }^{2} z_{q}^{2}}{2}+\frac{1}{3}\left(z_{p}^{2}+z_{q}^{2}\right)+\frac{1}{9}\right) d z_{p} d z_{q} \\
& =2 m(p, q)^{2} \int_{0}^{1} \int_{0}^{z_{p}}\left(z_{p}^{2}-z_{p}\left(z_{p}^{2}+z_{q}{ }^{2}+\frac{2}{3}\right)\right) d z_{p} d z_{q} \\
& +m(p, q)^{2}\left(\frac{1}{20}+\frac{1}{20}+\frac{1}{18}+\frac{1}{9}+\frac{1}{9}+\frac{1}{9}\right) \\
& =2 m(p, q)^{2}\left(\frac{1}{4}-\frac{1}{5}-\frac{1}{15}-\frac{2}{9}\right)+m(p, q)^{2}\left(\frac{1}{10}+\frac{1}{18}+\frac{1}{3}\right)=\frac{m(p, q)^{2}}{90}
\end{aligned}
$$

Hence (24) is proved.

The interaction indices for the two-additive model are, using (4)

$$
\begin{aligned}
& I^{\mu}(k)=m(k)+\frac{m(k, \cdot)}{2} \\
& I^{\mu}(p, q)=m(p, q)
\end{aligned}
$$

There is a clear difference between the Sobol' and the interaction indices, due to the presence of the square in the definition of the Sobol' indices. We observe that $I^{\mu}(p, q)$ and $\operatorname{Var}\left[f_{p, q}^{\mathrm{Lo}}\right]$ are both proportional to $m(p, q)$ or its square. By contrast, $\operatorname{Var}\left[f_{k}^{\mathrm{Lo}}\right]$ is not proportional to the square of $I^{\mu}(k)$. Term $m(k)$ takes more importance in $\operatorname{Var}\left[f_{k}^{\mathrm{Lo}}\right]$.

The Sobol' and interaction indices are both based on sensitivity analysis, but performed in a different manner. The interaction indices consider the average value of the partial derivative of $f^{\mathrm{Lo}}$ w.r.t. its components (see (91)), and is thus the mean value of a local sensitivity analysis. By contrast, the Sobol' indices comes from a sensitivity analysis based on variance. They are 
used for instance to identify which factors shall be fixed (the other variables being unknown and governed by uniform distribution) in order to reduce as much as possible the variance on the output variable.

It is not clear how to compare the interaction indices for singletons and pairs. For instance, if $I^{\mu}(1)=2 I^{\mu}(1,2)$, does it mean that the interaction between variables 1 and 2 is twice more important than the importance of criterion 1? Such a comparison is possible with the Sobol' indices as the variance of the output variable is decomposed into the variance of each variable individually, each pair of variables, and so on. This allows to compare the Sobol' indices for different terms. Then if $\operatorname{Var}\left[f_{1}^{\mathrm{Lo}}\right]=2 \operatorname{Var}\left[f_{1,2}^{\mathrm{Lo}}\right]$, then one can say that variable 1 alone is twice more influential than the interaction between variables 1 and 2 .

\section{References}

[1] G. Choquet. Theory of capacities. Annales de l'Institut Fourier, 5:131-295, 1953.

[2] R. A. Fisher and W. A. Mackenzie. The manurial response of different potato varieties. $J$. of Agricultural Science, 13:311-320, 1923.

[3] M. Grabisch. $k$-order additive discrete fuzzy measures and their representation. Fuzzy Sets and Systems, 92:167-189, 1997.

[4] M. Grabisch and Ch. Labreuche. A decade of application of the Choquet and Sugeno integrals in multi-criteria decision aid. Annals of Operations Research, 175:247-286, 2010. doi: $10.1007 / \mathrm{s} 10479-009-0655-8$.

[5] M. Grabisch, J.-L. Marichal, and M. Roubens. Equivalent representations of set functions. Mathematics of Operations Research, 25(2):157-178, 2000.

[6] P. L. Hammer and S. Rudeanu. Boolean Methods in Operations Research and Related Areas. Springer, 1968.

[7] R. L. Keeney and H. Raiffa. Decision with Multiple Objectives. Wiley, New York, 1976.

[8] L. Lovász. Submodular functions and convexity. In A. Bachem, M. Grötschel, and B. Korte, editors, Mathematical programming. The state of the art, pages 235-257. Springer Verlag, 1983.

[9] G. Owen. Multilinear extensions of games. Management Sci., 18:64-79, 1972.

[10] G. C. Rota. On the foundations of combinatorial theory I. Theory of Möbius functions. Zeitschrift für Wahrscheinlichkeitstheorie und Verwandte Gebiete, 2:340-368, 1964.

[11] M. Roubens. Interaction between criteria and definition of weights in MCDA problems. In 44th Meeting of the European Working Group "Multicriteria Aid for Decisions", Brussels, Belgium, October 1996.

[12] I. M. Sobol'. On sensitivity estimation for nonlinear mathematical models. Matematicheskoe Modelirovanie, 2:112-118, 1990. (in russian).

[13] I. M. Sobol'. Sensitivity estimates for nonlinear mathematical models. Mathematical Modeling and Computational Experiment, 1:407-414, 1993.

[14] M. Sugeno. Theory of fuzzy integrals and its applications. PhD thesis, Tokyo Institute of Technology, 1974. 